\title{
Anglais de spécialité et logométrie. L'exemple des débats présidentiels américains
}

\section{Marion Bendinelli}

\section{(2) OpenEdition}

1 Journals

Édition électronique

URL : http://journals.openedition.org/asp/2533

DOI : 10.4000/asp.2533

ISSN : 2108-6354

\section{Éditeur}

Groupe d'étude et de recherche en anglais de spécialité

\section{Édition imprimée}

Date de publication : 1 novembre 2011

Pagination : 103-123

ISSN : 1246-8185

\section{Référence électronique}

Marion Bendinelli, «Anglais de spécialité et logométrie. L'exemple des débats présidentiels

américains », ASp [En ligne], 60 | 2011, mis en ligne le 07 octobre 2014, consulté le 02 novembre 2020. URL : http://journals.openedition.org/asp/2533 ; DOI : https://doi.org/10.4000/asp.2533

Ce document a été généré automatiquement le 2 novembre 2020.

Tous droits réservés 


\title{
Anglais de spécialité et logométrie. L'exemple des débats présidentiels américains
}

\author{
Marion Bendinelli
}

$1 \quad$ Les études portant sur les débats présidentiels américains se divisent principalement en deux catégories. Certaines rendent compte de leur évolution et retracent ainsi l'histoire des médias et de la vie politique américaine (Jamieson \& Birdsell 1988, Minow \& Lamay 2008). D'autres s'intéressent aux stratégies verbales et non verbales employées par les locuteurs (Friedenberg 1994, 1997 ; Dailey, Hinck \& Hinck 2008). Toutes néanmoins tâchent d'éclairer le fonctionnement de la communication politicomédiatique aux États-Unis. Notre démarche emprunte une autre voie, envisagée ailleurs (Rouveyrol 2004) mais originale pour l'étude des débats présidentiels américains : empruntant les outils et méthodes de la logométrie (Mayaffre 2004), nous entendons caractériser ces interactions dans la perspective définie en anglais de spécialité.

2 Dans le cadre de cet article, nous présentons tout d'abord notre corpus d'étude ainsi que le logiciel Hyperbase ${ }^{1}$ que nous utilisons. Des relevés lexicaux sont ensuite étudiés dans le but de souligner certaines spécificités des débats. Nous nous interrogeons en sections 2 et 3 sur ce que ces relevés peuvent révéler du cadre interactionnel. En section 4, le modèle multidimensionnel de Paul A. Chilton (2004) est introduit afin de proposer une modélisation possible du contenu lexical et de la structuration cognitive du corpus dans son ensemble. En section 5, la question du genre discursif est traitée au travers des travaux de Norman Fairclough (1994, 1995, sqq.), lesquels portent notamment sur les discours politiques et journalistiques médiatisés. Avant d'envisager le résultat des analyses menées, il convient de préciser la place du discours politique et des débats présidentiels américains au sein du champ de recherche en anglais de spécialité. 


\section{Postulats théoriques, corpus et méthodologie}

\subsection{Discours politique, débats présidentiels et anglais de spécialité}

3 Tout acte de communication se définit selon Patrick Charaudeau (2002) par un contrat engageant des partenaires impliqués de manière plus ou moins active dans l'interaction. L'interprétation de leurs dires et de leurs comportements respectifs suppose d'identifier ces partenaires et de connaître les instances qu'ils représentent. Par «instance», P. Charaudeau désigne une entité humaine abstraite douée d'une intentionnalité et catégorisée en fonction de ses attributs identitaires, des rôles et de la place qui lui sont assignés dans et par le dispositif de communication (2005: 42). Toute pratique suppose l'existence d'une ou plusieurs instance(s); le domaine politique n'échappant pas à la règle, $\mathrm{P}$. Charaudeau dit à son sujet :

[I]l semble possible de le [= le domaine politique] déterminer quand sont traitées simultanément, et en interaction, les questions de l'action politique, de sa finalité et de son organisation; des instances qui sont parties prenantes dans cette action; des valeurs au nom desquelles est menée cette action. (2005: 12 - en italique dans le texte).

D'après l'auteur, les instances impliquées dans « l'action politique » sont les instances politique, citoyenne et médiatique. La première agit tout à la fois au nom des citoyens (desquels elle tire sa légitimité) et pour eux («en vue de l'obtention d'un bien commun ", Charaudeau 2005: 12); l'instance médiatique se fait le relais - tantôt didactique, tantôt critique - de la parole politique et citoyenne. L'expression de l'instance politique se fait, en raison de ses prérogatives et des relations qu'elle entretient avec les deux autres instances, en trois espaces dont les frontières ne sont pas hermétiques: il s'agit des espaces de discussion, d'action et de persuasion (Charaudeau 2005: 16). L'étude du domaine politique ne peut faire l'économie de l'étude de la parole de ses acteurs, car, s'il est besoin de le rappeler, « le discours est constitutif du politique » (Trognon \& Larrue 1994: 10), il est «intrinsèquement lié à l'organisation de la vie sociale»(Charaudeau 2005: 32) et constitue «le lieu d'engagement du sujet, de justification de son positionnement et d'influence de l'autre » (ibid.). Le discours politique apparaît ainsi comme une activité à part entière, constituée par et pour le social, se singularisant par ses composantes technique et intellectuelle, son triple questionnement (sur l'action, les instances et les valeurs qui le guident) et sa matérialisation en trois espaces (de discussion, d'action et de persuasion). Il peut prendre diverses formes variant selon le dispositif de communication et la situation d'énonciation rencontrés: les meetings, débats, entretiens, professions de foi, interventions en Parlement, etc., sont tout autant d'instanciations concrètes du discours politique ${ }^{2}$.

5 Peut-on définir le domaine politique comme un milieu ? Selon Marie-Christine Deyrich, un milieu est «un ensemble d'éléments d'ordre naturel et d'éléments sociaux, des infrastructures produites par les hommes, des institutions, des cultures, etc., le tout formant un système. » $(2004: 125)$. Le domaine politique satisfait aux critères de cette définition compte tenu de son organisation interne (articulation de diverses institutions étatiques, conseils et commissions qui tout à la fois légitiment et résultent de l'action politique) et de l'existence d'acteurs bien identifiés (membres de la classe politique, etc.) qui ont à maîtriser certains codes, notamment idéologiques et culturels. Plus particulièrement, à la suite de Michel Petit (2004), nous définissons le milieu 
politique comme un milieu spécialisé parce qu'il suppose l'exercice d'une activité structurée, reconnue socialement mais non strictement professionnelle ${ }^{3}$. La nature "spécialisée » du milieu politique résulte plus généralement du domaine d'activité dans lequel ses acteurs sont amenés à agir (Petit 2010: 10). En effet, le domaine politique, défini, nous l'avons vu, comme un secteur structuré, socialement construit et occupant une place identifiable au sein de la société forme bien un domaine spécialisé au sens donné par M. Petit :

[N]ous appellerons domaine spécialisé tout secteur de la société constitué autour et en vue de l'exercice d'une activité principale qui, par sa nature, sa finalité et ses modalités particulières ainsi que par les compétences particulières qu'elle met en jeu chez ses acteurs, définit la place reconnaissable de ce secteur au sein de la société et d'un ensemble de ses autres secteurs et détermine sa composition et son organisation spécifiques. (2010: 9).

Les discours qui sous-tendent l'existence d'un tel domaine sont à leur tour considérés comme "spécialisés", ce statut se transmettant de fait du domaine aux moyens linguistiques, discursifs, rhétoriques, terminologiques qui le caractérisent.

Dans un tel cadre, le discours politique anglophone, et à travers lui, les débats présidentiels américains, peuvent être envisagés comme un anglais de spécialité en ce que l'anglais qui y est employé est «l'expression d'un domaine spécialisé dans cette langue » (SAES 2011: 3). À ce titre, l'étude des débats présidentiels s'inscrit dans le champ de l'anglais de spécialité, discipline que M. Petit définit comme :

[L]a branche de l'anglistique qui traite de la langue, du discours et de la culture des communautés ou milieux professionnels et autres groupes sociaux spécialisés anglophones ainsi que de l'enseignement de cet objet, et qui constitue cet enseignement. (2004:7)

Les analyses logométriques menées s'intéressent en particulier à la structuration discursive des débats présidentiels et à la manière dont elle reflète le cadre communicationnel et situationnel régulant les échanges. Nous nous interrogeons également sur ce que ces analyses peuvent révéler des stratégies énonciatives adoptées par les candidats. Pour ce faire, un corpus d'étude a été constitué.

\subsection{Les débats : corpus d'étude et implémentation informatique}

9 Le corpus d'étude se compose des vingt-deux face-à-face présidentiels à deux candidats $^{4}$ organisés depuis leur création, en 1960. Ces face-à-face présentent trois scénographies différentes - "conférence de presse", "rencontre avec un panel de citoyens » et "conversation avec le modérateur $»^{5}$ - et leur durée varie de soixante à quatre-vingt-dix minutes. Les interventions des candidats ayant pris part aux années électorales qui nous intéressent $(1960,1976,1984,1988,1996,2000,2004,2008)$ ont été retranscrites à partir du matériel diffusé par la commission en charge de l'organisation des débats (Commission on Presidential Debates) et par diverses archives audiovisuelles. Des informations relatives au canal non verbal ont été ajoutées aux retranscriptions; le tableau 1 en précise la nature et indique les codes adoptés. 
Tableau 1. Données supplémentaires incluses dans les retranscriptions des interventions des candidats

\begin{tabular}{|c|c|}
\hline Données supplémentaires encodées & Symbole \\
\hline $\begin{array}{l}\text { Pauses silencieuses ou vocalisées (correspondant à \& er } * \text {, *erm } * \text { ), } \\
\text { hésitations du locuteur sur des mots qu'il peine à prononcer }\end{array}$ & $<->$ \\
\hline $\begin{array}{l}\text { Reprises } \\
\text { - supposant la correction (avec conservation) de I'orientation } \\
\text { argumentative des propos tenus par le locuteur } \\
\text { - générées par le candidat lui-même ou provoquées par un autre } \\
\text { participant (adversaire, journaliste, modérateur ou membre de } \\
\text { I'auditoire) }\end{array}$ & $<@>$ \\
\hline $\begin{array}{l}\text { Interruptions } \\
\text { - modifiant I'orientation argumentative des propos tenus par le } \\
\text { locuteur } \\
\text { - générées par le candidat lui-même ou provoquées par un autre } \\
\text { participant (adversaire, journaliste, modérateur ou membre de } \\
\text { l'auditoire) }\end{array}$ & $<\#>$ \\
\hline
\end{tabular}

Le corpus, dont la taille est de 317270 occurrences, a été découpé par année électorale (1960, [...] 2008) et par candidat (Nixon, Kennedy, [...] McCain, Obama) ; il est ainsi formé de seize partitions. Afin de naviguer dans les interventions tout en dépassant le processus de lecture naturelle, nous inscrivons notre démarche dans le cadre de la logométrie, une analyse de discours automatisée au moyen de logiciels de traitement documentaire et statistique, ici, le logiciel Hyperbase.

11 Bien qu'il s'agisse de retranscriptions de données orales, les fichiers incluent des marques de ponctuation de l'écrit (point, virgule, point d'interrogation) afin que le logiciel puisse procéder au découpage en phrases et en paragraphes, étape nécessaire à la phase de traitement statistique. Les divers calculs effectués (calculs de fréquence, de co-occurrence, etc.) déconstruisent le corpus pour en souligner l'homogénéité et l'hétérogénéité aux niveaux lexical (formes et lemmes ${ }^{6}$ ), grammatical, syntaxique, thématique et sur le plan des réseaux de co-occurrents. Les relevés et représentations graphiques générés par le logiciel contribuent à déterminer quelles variables linguistiques sont révélatrices de caractéristiques discursives, rhétoriques, génériques définissant le corpus et/ou certaines de ses partitions. On le voit, la logométrie permet de mener des analyses multi-niveaux et d'identifier des spécificités qu'une méthode d'analyse manuelle ne pourrait identifier.

12 La question se pose de savoir par quel angle aborder le corpus d'étude. La logométrie propose divers parcours interprétatifs selon les requêtes soumises au logiciel et les fonctions activées. Nous avons fait le choix d'utiliser ici la fonction DISTRIBUTION d' Hyperbase afin d'envisager le contenu grammatical et lexical du corpus. Nous nous intéressons plus précisément à ses hautes fréquences lemmatisées pour avoir une vision globale des types lexicaux les plus employés dans les débats. Avant d'examiner des structurations plus complexes (relatives à l'organisation cognitive et générique), il s'agit tout d'abord de s'interroger sur ce que le lexique le plus fréquent du corpus peut révéler des pratiques discursives des candidats. 


\section{2. Étude des hautes fréquences lexicales des débats}

13 Les hautes fréquences lemmatisées permettent d'identifier et d'analyser ce que Jules Duchastel et Victor Armony nomment «stabilité intertextuelle (1993:4), c'est-à-dire le vocabulaire caractéristique d'un événement de communication, les mots «obligés, ceux qu'aucun locuteur ne peut conjurer à l'intérieur d'un genre ou dans un contexte déterminé» (ibid.). Pour évaluer et établir la représentativité des lemmes relevés dans le tableau 2, les fréquences observées sont comparées à celles caractérisant l'ensemble du corpus COCA (Corpus of Contemporary American English) ainsi qu'aux données de Douglas Biber, Stig Johansson, Geoffrey Leech et alii (1999) pour les registres ÉCRITS SCIENTIFIQUES, INFORMATION, FICTION et CONVERSATION.

\subsection{Lexiques commun et spécifique}

Au-delà des contraintes linguistiques qui apparaissent dans les premiers rangs ${ }^{7} \mathrm{du}$ tableau 2, on note tout d'abord que les parties du discours se répartissent de manière semblable à celles observées dans COCA : parmi les 50 plus hautes fréquences, on retrouve des prépositions, des conjonctions, des verbes et des pronoms, tandis que les déterminants (hors articles), les substantifs et les adjectifs apparaissent davantage dans les 50 rangs suivants. La distribution des catégories grammaticales au sein des débats présidentiels reflète ainsi celle de l'anglais américain contemporain, en dehors de toute distinction de registre.

Une seconde comparaison portant plus particulièrement sur les rangs de fréquence amène à classer les substantifs en deux catégories. D'une part, on retrouve les substantifs dont les rangs de fréquence sont assez proches de ceux observés dans COCA : c'est le cas des lemmes year, time, thing, way ${ }^{8}$. Cette première catégorie regroupe des substantifs appartenant au langage quotidien oral et constitue à ce titre un lexique que l'on peut qualifier de « commun ». On distingue d'autre part des substantifs dont la fréquence d'emploi relative dans notre corpus est fortement supérieure à celle observée dans COCA : il s'agit des lemmes people, president, country, tax, senator, world, government, dollar et America ${ }^{9}$. Cette seconde catégorie forme un lexique dit "spécifique » en ce que les substantifs qui le composent sont tantôt liés au contexte du débat et à l'échéance électorale, tantôt à la vie politique et à l'exercice du pouvoir.

Tableau 2. Relevé des hautes fréquences du corpus

\begin{tabular}{|c|c|l|r|r|l|l|l|l|}
\hline $\begin{array}{c}\text { Rang de } \\
\text { fréquence }\end{array}$ & $\begin{array}{c}\text { Nombre } \\
\text { d'occurrences }\end{array}$ & \multicolumn{2}{|c|}{$\begin{array}{c}\text { Lemmes / } \\
\text { Ponctuation }\end{array}$} & \multicolumn{5}{|l|}{} \\
\hline \hline 1 & 16630 &, & 35 & 1258 & $\begin{array}{l}\text { can (modal } \\
\text { aux.) }\end{array}$ & 68 & 613 & time \\
\hline 2 & 15499 &. & 36 & 1206 & make & 69 & 605 & one \\
\hline 3 & 14882 & the & 37 & 1205 & president & 70 & 600 & from \\
\hline 4 & 13711 & be & 38 & 1194 & there & 71 & 598 & out \\
\hline
\end{tabular}




\begin{tabular}{|c|c|c|c|c|c|c|c|c|}
\hline 5 & 8505 & and & 39 & 1187 & what & 72 & 595 & America \\
\hline 6 & 8347 & to & 40 & 1173 & would & 73 & 579 & believe \\
\hline 7 & 7745 & I & 41 & 1170 & get & 74 & 573 & which \\
\hline 8 & 6840 & of & 42 & 1050 & if & 75 & 572 & take \\
\hline 9 & 6006 & we & 43 & 989 & as & 76 & 562 & up \\
\hline 10 & 5877 & $a$ & 44 & 950 & now & 77 & 560 & those \\
\hline 11 & 5531 & in & 45 & 905 & my & 78 & 545 & some \\
\hline 12 & 5381 & have & 46 & 888 & year & 79 & 534 & united \\
\hline 13 & 4003 & $\begin{array}{l}\text { that } \\
\text { (complementizer + } \\
\text { relativizer) }\end{array}$ & 47 & 887 & who & 80 & 534 & all \\
\hline 14 & 3844 & it & 48 & 865 & want & 81 & 534 & $?$ \\
\hline 15 & 3634 & not & 49 & 859 & know & 82 & 525 & dollar \\
\hline 16 & 3271 & do & 50 & 838 & so & 83 & 522 & senator \\
\hline 17 & 2794 & you & 51 & 828 & or & 84 & 512 & thing \\
\hline 18 & 2515 & for & 52 & 809 & at & 85 & 508 & very \\
\hline 19 & 2199 & & 53 & 777 & about & 86 & 499 & well \\
\hline 20 & 1911 & this & 54 & 746 & because & 87 & 498 & let \\
\hline 21 & 1846 & go & 55 & 726 & an & 88 & 495 & states \\
\hline 22 & 1797 & $\begin{array}{l}\text { that } \\
\text { (demonstrative } \\
\text { pronoun) }\end{array}$ & 56 & 725 & when & 89 & 482 & world \\
\hline 23 & 1788 & $\begin{array}{l}\text { that } \\
\text { (démonstrative } \\
\text { determiner) }\end{array}$ & 57 & 720 & country & 90 & 481 & work (verb) \\
\hline 24 & 1698 & our & 58 & 711 & have to & 91 & 480 & his \\
\hline 25 & 1681 & on & 59 & 707 & $\begin{array}{l}\operatorname{tax} \\
\text { (substantive) }\end{array}$ & 92 & 468 & way \\
\hline 26 & 1619 & he & 60 & 702 & us & 93 & 463 & 's (genitive) \\
\hline 27 & 1605 & with & 61 & 675 & @ & 94 & 460 & your \\
\hline
\end{tabular}




\begin{tabular}{|c|c|l|c|l|l|l|l|l|}
\hline 28 & 1541 & think & 62 & 669 & by & 95 & 460 & these \\
\hline 29 & 1538 & but & 63 & 660 & good & 96 & 459 & give \\
\hline 30 & 1451 & will (modal aux.) & 64 & 655 & me & 97 & 455 & should \\
\hline 31 & 1437 & they & 65 & 653 & just & 98 & 451 & more \\
\hline 32 & 1402 & people & 66 & 640 & them & 99 & 448 & government \\
\hline 33 & 1328 & say (verb) & 67 & 624 & their & 100 & 444 & than \\
\hline 34 & 1301 & @card@ & & & & \\
\hline
\end{tabular}

La coexistence de ces deux lexiques se voit plus généralement confirmée par le dictionnaire de l'ensemble des formes graphiques employées dans le corpus et par la liste des phrases clés ${ }^{10}$ des locuteurs. Les trois énoncés qui suivent illustrent cette imbrication.

The basic decision of the Supreme Court is that each person has to make this judgement in her own life, and that's the way it's been done. (W. Mondale, 1984)

How can Barry Goldwater sitting in Arizona know whether I'm talking on the issues or not, when we put out position paper after position paper, he puts out position paper after position paper, and we see this much about it, because everyone else is fascinated with polls and who's up or down today and who's going to be up or down tomorrow. (G. H. Bush, 1988)

First of all, it's a balanced budget amendment to the Constitution which President Clinton defeated. He twisted arms and got six democrats to vote the other way but we lost by one vote. (R. Dole, 1996)

Walter Mondale, George H. Bush et Robert Dole s'expriment respectivement au sujet d'une décision judiciaire, de leur programme et d'un amendement rejeté par le Congrès. Le vocabulaire technique/spécifique - Supreme Court, position paper, polls, budget amendment, Constitution - côtoie des expressions plus communes, telles que basic decision, that's the way it is, to see, to be up/down, to twist arms, [to vote] the other way. Il apparaît ainsi que, comme tout discours spécialisé, le discours politique, vu au travers des débats présidentiels, suppose de maîtriser un langage commun et un lexique spécifique.

\subsection{Débats et registre conversationnel}

Le tableau 2 indique par ailleurs que les candidats engagés dans des débats présidentiels tendent à emprunter les codes discursifs des échanges de type dialogal. En effet, les proportions de chaque catégorie grammaticale (quantité de substantifs, de pronoms, de verbes, d'adverbes, etc.) sont proches de celles relevées par D. Biber et alii pour le registre conversationnel $(1999: 65,92)$. Plus particulièrement, en ce qui concerne les marqueurs discursifs employés, on note la haute fréquence des pronoms de l'interlocution I et we (occupant respectivement les rangs 7 et 9) et de la négation not (15 rang), des unités caractéristiques, dans COCA comme chez D. Biber et alii (1999: $150 ; 259)$, des productions orales ${ }^{11}$. 
19 La prégnance de ces marqueurs dialogaux témoigne de la nature intrinsèquement interactive des débats qui, comme les conversations, "invitent [les participants] à l'expression de l'accord et du désaccord $»^{12}$ (Biber et alii 1999 : 159). En conséquence, les débats présidentiels semblent partager certaines propriétés $d u$ registre conversationnel, un registre qui se définit comme une variété «orale, directement interactive [...], probablement la forme de communication humaine la plus basique $»^{13}$ (Biber et alii 1999: 16) ayant pour objet « les vies et les intérêts des interlocuteurs [qui partagent] le même contexte physique et spatio-temporel $»^{14}(i b i d$.) et un même ensemble de connaissances personnelles. Quelles autres traces témoignent de cette proximité ?

La présence des symboles «- 》 (pauses vocalisées et hésitations - 19 ${ }^{\mathrm{e}}$ rang) et " @ 》 (reprises $-61^{\mathrm{e}}$ rang) indique que la parole politique, dans les débats, est saccadée et non linéaire. Que cette caractéristique discursive relève ou non d'une stratégie consciente visant à désacraliser l'aspect (trop) formel des débats ${ }^{15}$, elle se révèle intéressante pour démystifier la prise de parole des hommes politiques. De plus, elle attire notre attention sur le fait que les candidats tendent à élaborer leur dire en même temps qu'ils le formulent : c'est ce que D. Biber et alii appellent « production on line » $(1999: 1066)$. À ce titre, les débats apparaissent proches des conversations.

21 Les données quantifiées symbolisées par @card@ (34 ${ }^{\mathrm{e}}$ rang) et le lemme dollar (82 rang) sont un autre élément à prendre en compte. Quantifier ses dires pour décrire des faits de la façon la plus précise et concrète possible est souvent considéré, notamment par les hommes politiques, comme un moyen discursif permettant de paraître compétent, légitime et crédible. Jimmy Carter et Gerald Ford (1976) sont particulièrement représentatifs de ce type de parole :

We've got 500,000 more Americans out of jobs today than were out of work three months ago and, since Mr Ford's been in office two years, we've had a 50 percent increase in unemployment from 5 million people out of work to 2 and a half million more people out of work and a total of 7 and a half million. (J. Carter, 1976)

And I recommended that to the Congress with a budget ceiling of 395 billion dollars, and that would have permitted us to have a 25 billion dollar tax reduction. In my tax reduction program for middle-income taxpayers, I recommended that the Congress increase personal exemptions from 750 dollars per person to 1,000 dollars per person. (G. Ford, 1976)

Pour D. Biber et alii, l'acte de quantifier est un trait définitoire du registre conversationnel du fait qu'il convient d'y rapporter des faits ou de construire un objet de discours en le(s) chiffrant et/ou en le(s) situant dans le temps et/ou l'espace (1999: 279). Par conséquent, l'importance de ces données dans notre corpus d'étude constitue un nouvel argument en faveur de la proximité établie entre débats présidentiels et registre conversationnel.

\section{3. Étude des pronoms}

La présence des pronoms personnels (employés en position sujet et complément) et possessifs parmi les hautes fréquences du corpus est telle qu'il parait intéressant de les étudier plus en détail. Quelles informations peuvent-ils apporter quant au cadre interactionnel? 


\subsection{Distribution dans le corpus}

24 Le paradigme des pronoms est complet à l'exception des pronoms féminins she et her. Cette absence n'est pas étonnante, car les référents féminins sont (encore) rares dans la vie politique et institutionnelle du pays ainsi que dans le monde des affaires. Seul le milieu journalistique semble y faire exception. Dans les débats, il n'est question de femmes que dans les cas où les candidats s'adressent à une journaliste (Andrea Mitchell), parlent de leurs filles ou de leur épouse, de mères de soldats partis en guerre ou de jeunes filles rencontrant des problèmes dans l'enceinte de l'école: le renvoi à des référents féminins est donc ponctuel, principalement lié à des thématiques bien précises et destiné à émouvoir l'auditoire.

Les pronoms (personnels et possessifs) de l'interlocution (I, we, you et corrélats) sont employés de manière majoritaire dans le corpus (20 310 occurrences) : cette fréquence laisse supposer que la relation entre locuteur-candidat et interlocuteur est prégnante. De quel interlocuteur s'agit-il ? À qui s'adressent les candidats?

Tout événement de communication de type interview médiatisé (reposant sur des échanges questions-réponses entre modérateur/journaliste et invité politique) se caractérise par un double destinataire (voir notamment Vion 1992, Bendinelli 2008) : l'intervieweur (le modérateur et/ou les journalistes) en est le destinataire explicite, l'auditoire (public en salle, téléspectateurs, etc.) et le candidat-adversaire, les destinataires implicites. Catherine Kerbrat-Orecchioni (1990) définit cette situation de double destinataire comme relevant d'un trope communicationnel. Cette désignation ne se révèle cependant pas pertinente pour décrire les débats présidentiels car les destinataires implicites ne sont pas «relativement camouflé[s]» (Kerbrat-Orecchioni 1990 : 96). En effet, non seulement les candidats multiplient les parallèles avec leur adversaire au moyen d'attaques principalement explicites (Dailey et alii 2008 : 18-19), mais l'auditoire aussi est considéré par tous comme la véritable cible des échanges. Son existence physique n'est pas ignorée, contrairement à ce qui se passe dans le cas de tropes: la scénographie (les candidats font face au public et aux caméras), les propos tenus en ouverture de débat par le modérateur, ou encore les adresses directes que s'autorisent certains hommes politiques (telles que «Ladies and gentlemen " prononcé à plusieurs reprises par John Kerry en 2004) sont autant d'indices prouvant que le public est l'une des cibles explicites des candidats. Dans les débats, la relation I-we/you est donc évidente et apparemment mise au premier plan.

La situation est en réalité plus complexe car les pronoms (personnels et possessifs) de la délocution (he, it, they + corrélats) - qui renvoient à des référents extérieurs au cadre communicationnel - sont plus fréquemment employés que les pronoms de deuxième personne you et your ${ }^{16}$. S'il est donc vrai que l'auditoire est le destinataire réel des hommes politiques en débat, ces chiffres indiquent qu'il n'est pourtant pas celui que les candidats interpellent majoritairement, ceux-ci préférant convoquer dans leurs dires tout un ensemble de référents externes. De ce fait, la hiérarchie entre les divers acteurs du débat - locuteur, interlocuteur et référents externes - est bouleversée. Ce n'est pas tant la relation dialogale entre candidats et auditoire qui est privilégiée, que la relation entre candidats, personnes tierces et faits. 


\subsection{Un dialogue fictif}

28 participants (le modérateur, les journalistes et l'auditoire) apparaît comme une fiction dans la mesure où la relation qui compte vraiment est celle qui les unit aux référents externes. Nous nommons dialogue fictif cette situation d'énonciation particulière que les candidats, engagés dans un débat présidentiel, créent au cours des échanges. Dans ce dialogue fictif, le locuteur s'octroie le rôle central et convoque des référents externes qu'il décrit et juge tout en s'adressant à l'auditoire, destinataire réel sur le plan communicationnel mais périphérique sur le plan linguistique.

29

analyse vient-elle infirmer le précédent rapprochement observé entre débat et registre conversationnel? Cela ne semble pas être le cas, car le dialogue fictif ici dessiné confirme que les débats adoptent les codes de la conversation (relation primaire $I / y o u$ ) et sont bien l'occasion pour les candidats de parler d'événements externes dont dépendent les "vies et intérêts des interlocuteurs " (Biber et alii 1999 :

16). Nous illustrons cette mise en scène par deux énoncés tirés du corpus :

But, where I differ with my opponent is \# I am not going to make unilateral cuts in our strategic defense systems or support some freeze when they have superiority. (G. H. Bush, 1988)

First of all, what my opponent wants you to forget is that he voted to authorize the use of force and now says "it's the wrong war, at the wrong time, at the wrong place". (G. W. Bush, 2004)

George Bush père et fils désignent leurs adversaires (respectivement Michael Dukakis et John Kerry) par un syntagme nominal my opponent ; ils convoquent en discours des référents externes (l'Union Soviétique désignée par they chez Bush père) ou présentés comme tels (le candidat démocrate John Kerry désigné par he chez Bush fils). Ils s'opposent assez explicitement aux propos et/ou propositions de leurs adversaires démocrates qu'ils incluent pourtant au plan délocutoire. La position centrale est occupée par les deux candidats républicains, Bush père assumant pleinement ses propos (recours à I), Bush fils se positionnant davantage comme porte-parole d'un groupe plus ou moins explicitement défini (recours à our). Les référents externes sont majoritaires et la visée argumentative est bien d'influencer les connaissances de l'auditoire qui n'est pourtant désigné explicitement qu'une seule fois (you chez George W. Bush).

\section{Débats et espaces représentationnels}

31 L'étude du lexique appelle un autre type d'analyse. En effet, les substantifs relevés dans le tableau 2 renvoient principalement à des référents animés (people, president), spatiaux (United States, America, world), temporels (now, year, time)et, de manière moins marquée, à des thématiques (tax, dollar). À ce titre, il est possible de mettre en œuvre les outils de la Discourse Space Theory de P. A. Chilton (2004) et d'exploiter le modèle multidimensionnel qu'il propose. 


\subsection{Présentation}

S'inspirant de la notion d'espaces mentaux de Gilles Fauconnier (1985), le modèle multidimensionnel de P. A. Chilton permet de décrire une production verbale au moyen d'une représentation (figure 1) articulée autour de quatre axes. Sur ces axes ou espaces subjectif, géographique, temporel et modal, les référents (concrets ou non, réels ou fictifs, animés ou inanimés, etc.) et expressions modales employés prennent tour à tour place ${ }^{17}$.

La position respective des items lexicaux est définie en fonction de la distance qu'ils sous-tendent vis-à-vis du centre déictique (Vershueren 1999 : 20), élément essentiel du modèle, car correspondant à la place occupée par le locuteur. Plus un référent est proche du centre déictique, plus il est jugé bon, bénéfique, souhaitable, et son existence est considérée comme proche dans le temps et dans l'espace; inversement, plus le référent en est distant, plus il est considéré comme lointain (dans le temps et l'espace), incertain et nuisible.

Figure 1. Le modèle adapté des espaces représentationnels de P. A. Chilton

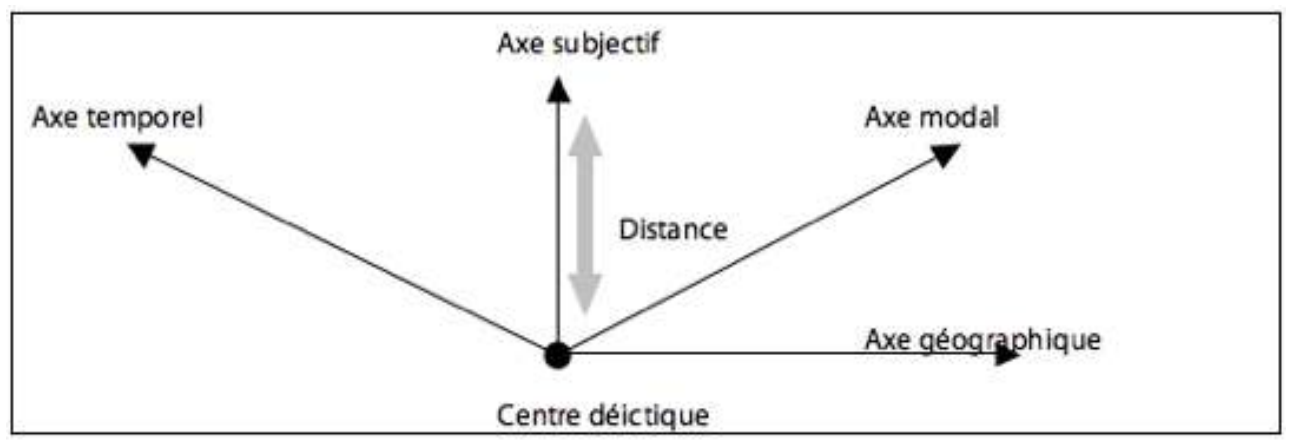

Les espaces représentationnels donnent une clef de lecture originale aux prises de parole qui les sous-tendent, car ils permettent de "voir " le monde que le locuteur construit etles relations qu'il entretient avec les éléments qui le composent. Chemin faisant, ils offrent la possibilité d'identifier une partie des connaissances convoquées lors de l'interprétation: en ce sens, les espaces reconstruisent des "cadres cognitifs " (Chilton 2004 : 56). Une telle représentation spatiale permet également de dépasser la lecture linéaire des prises de parole qui la sous-tendent : la figure obtenue reconstruit un prototype des productions prises en compte, elle en subsume les éléments les plus représentatifs, indépendamment des variations thématiques. Dans ce cadre, en quoi consiste la représentation spatiale des débats présidentiels? Quelles conclusions en tirer?

\subsection{Espaces représentationnels des débats présidentiels}

35 À partir du lexique le plus fréquent, on élabore la représentation spatiale suivante (figure 2). 
Figure 2. Espaces représentationnels des débats présidentiels médiatisés (hors axe modal ${ }^{18}$ )

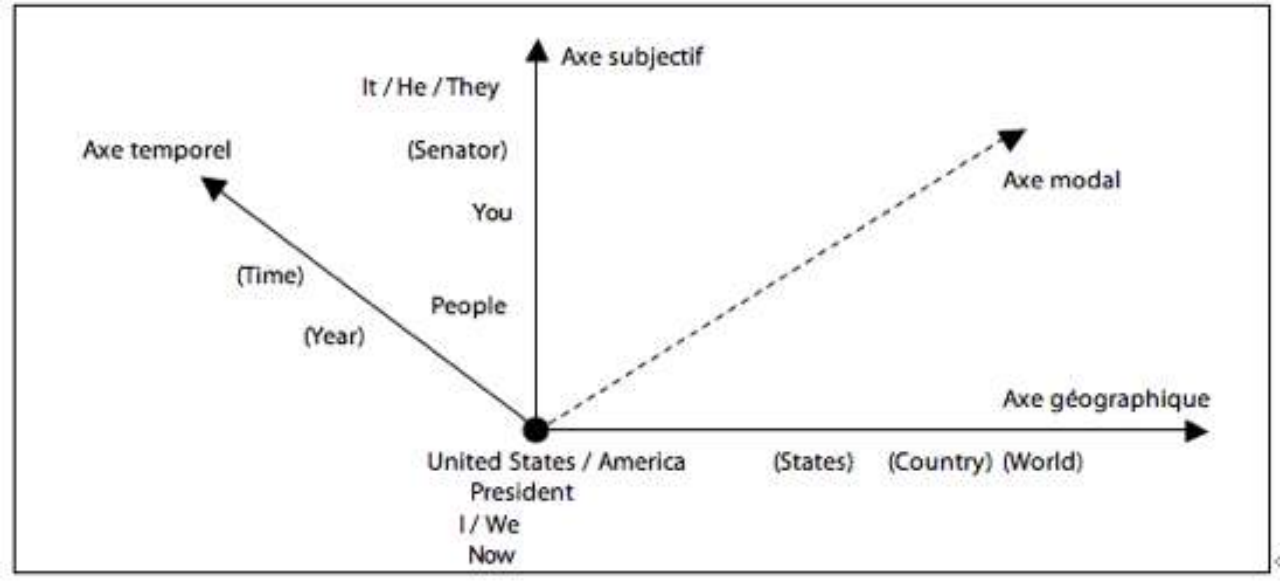
celle qu'occupent les candidats c'est-à-dire la position dite « ego, hic et nunc ». En effet, pour chaque espace, un référent (ou plus) peut être positionné sur le centre déictique : les pronoms I et we, l'adverbe now, les substantifs president, America, United States. De plus, l'axe subjectif semble être l'espace représentationnel le plus complet: on y dénombre le plus grand nombre de référents. En comparaison, les axes temporels et géographiques apparaissent secondaires : les référents à placer sur ces espaces sont peu nombreux et renvoient à des entités vagues, relativement mal définies, à l'image de time, country et world.

Une telle configuration des espaces représentationnels est cohérente avec le dispositif de communication et le cadre énonciatif sous-jacents aux enjeux électoraux caractérisant les débats. La position « ego, hic et nunc » est en effet plus développée que d'autres parce que les candidats doivent mettre l'accent sur leur adéquation avec le statut de Président des États-Unis d'Amérique et sur les actions qu'ils pourront accomplir en tant que tel pour servir les intérêts de leur pays. L'axe subjectif ici modélisé fait également écho aux travaux de Kathleen. H. Jamieson et David. Birdsell (1988). Selon ces deux auteurs, les candidats cherchent en effet à contourner l'extrême rigidité des règles encadrant le déroulement des échanges en (re)mettant au premier plan leur propre subjectivité. Une telle posture favorise la comparaison entre prétendants et amène l'auditoire à faire un choix. Elle explique également pourquoi les axes spatio-temporels semblent mis au second plan. La confrontation (entre personnes et/ou entre personnes et événements) étant l'un des aspects centraux des débats, les candidats délaissent volontiers les espaces géographique et temporel pour mettre l'accent sur leur personne: ils sont décidés à faire part de leurs positionnements respectifs et intéressés par la comparaison systématique de leurs opinions et savoirs avec ceux de leur adversaire.

Il apparaît ainsi que, dans les débats comme dans les cas de conversations, les référents spatio-temporels sont marginalisés à la périphérie du cadre cognitif, alors que la subjectivité des locuteurs y occupe la place centrale. Le parallèle établi précédemment entre débats et registre conversationnel se voit ainsi confirmé sur les plans énonciatif et cognitif. 


\section{Un genre de discours hybride}

39 Il s'agit à présent de s'intéresser aux spécificités lexicales des discours des candidats (fonction sPÉCIFICITÉs d'Hyperbase), c'est-à-dire aux unités lexicales qui caractérisent en propre les dires d'un candidat et qui le distinguent des autres locuteurs. Un tel calcul permet d'évacuer la question des contraintes (notamment morphosyntaxiques) liées à la langue pour étudier les stratégies adoptées et les thématiques abordées. Nous allons montrer que ces spécificités peuvent contribuer à traiter de la question du genre de discours, telle qu'elle est envisagée dans le cadre de la Critical Discourse Analysis (ou $(\mathrm{CDA})^{19}$, modèle théorique né dans les années 1980 en Angleterre à la suite des travaux de Norman Fairclough.

\subsection{La CDA et la notion de genre : une brève présentation}

40 Reprenant un postulat formulé en ethnolinguistique au début du $\mathrm{XX}^{\mathrm{e}}$ siècle et dans les années 1980 par Pierre Bourdieu (1982), la CDA estime que l'utilisation de la langue structure la société tout autant qu'elle est structurée par elle (Fairclough 1995 : 54-55). Cette dynamique analytique se retrouve à plusieurs niveaux de la conceptualisation, notamment dans les définitions des notions de discours et de genre.

41 L'étude d'un discours suppose d'envisager les moyens d'expression d'une pratique sociale donnée et la manière dont celle-ci ou l'un de ses domaines se construit. Pour N. Fairclough, une pratique se définit comme l'activité d'un milieu (spécialisé ou non), traversé, bien que de manière souvent invisible (2001 : 233-4), par un ensemble d'idées, de valeurs et de croyances. L'utilisation de la langue se trouve ainsi contrainte par des conditions sociales, externes; elle l'est également par le genre dans lequel s'inscrit l'activité envisagée. En effet, pour N. Fairclough, la notion de genre se définit comme «l'utilisation de la langue telle qu'elle est associée à une pratique particulière tout en formant une partie de celle-ci $»^{20}(1995: 56)$. Un genre résulte de conventions stabilisées - ce qui en assure la reconnaissance et la reproduction - qui peuvent néanmoins évoluer conjointement à la/aux pratique(s) sociale(s) dont elles dépendent. Il implique en outre l'existence d'un processus de production préétabli (qu'il contribue aussi à élaborer) ainsi que la mise en œuvre de ressources de structuration et de mise en texte ${ }^{21}$ (Fairclough 2000:14). Un genre, associé à un discours, forme un type de discours. L'ensemble des types discursifs mis à l'œuvre dans un domaine social et/ou institutionnel compose l'ordre de discours de ce domaine ${ }^{22}$.

Dans le cadre de la CDA, les débats présidentiels sont conçus comme un texte, un objet discursif et social dont la production dépend d'une activité sociale, l'exercice de la politique. Cette activité est observée ici dans le cadre d'un événement communicationnel précis, le " débat médiatisé en contexte électoral », événement qui se définit par des contraintes socioculturelles, institutionnelles et discursives. Compte tenu du fait que le genre de discours façonne la production du texte (tout autant qu'il est façonné par lui), on devrait pouvoir relever des traces de sa présence dans les interventions des candidats. La question posée est double : comment saisir ces traces? Que révèlent-elles de la nature des débats? 


\subsection{Des spécificités lexicales des candidats au genre de discours hybride}

Les spécificités lexicales de tous les candidats ont été relevées et classées en neuf catégories en fonction de champs thématiques (catégories 1, 2, 3, 5) ou de fonctions pragmatiques (catégories 4, 6, 7, 8, 9) ; pour faciliter la lecture, seule une partie des formes relevées sont reproduites dans le tableau 3.

Tableau 3. Catégorisation thématique et/ou pragmatique des spécificités lexicales des candidats

\begin{tabular}{|c|c|c|}
\hline & Catégorie & Items lexicaux \\
\hline 1 & $\begin{array}{l}\text { Alliés ou ennemis } \\
\text { (noms propres et } \\
\text { communs) }\end{array}$ & $\begin{array}{l}\text { Eisenhower, Israel, enemy, opponent, terrorists/m, } \\
\text { Communist/m, freeze, Castro, Bin Laden, Soviets, Al } \\
\text { Qaida, Kim Il Jong, North Korea, Pakistan, Iran... . }\end{array}$ \\
\hline 2 & Lleux de conflits armés & $\begin{array}{l}\text { Vietnam, Quemoy and Matsu, Formosa Island, } \\
\text { Serbia, Iraq, Afghanistan... . }\end{array}$ \\
\hline 3 & $\begin{array}{l}\text { Valeurs et notions } \\
\text { abstraites fortement } \\
\text { connotées }\end{array}$ & $\begin{array}{l}\text { aid, faith, hero, ideal, freedom, passion, judgment, } \\
\text { light, promise, respect, liberty, secrecy, war, right... }\end{array}$ \\
\hline 4 & $\begin{array}{l}\text { Références } \\
\text { institutionnelles }\end{array}$ & $\begin{array}{l}\text { Washington, administration, agency, office, } \\
\text { convention, conference, congress, commission, bill, } \\
\text { court, policy ; Gingrich, Kemp, Brady... ; Dan } \\
\text { Quayle, Joe Lieberman, Sarah Palin. }\end{array}$ \\
\hline 5 & Programmation politique & scheme, program, package, reform, proposal, plan... \\
\hline 6 & $\begin{array}{l}\text { Communautés, groupes } \\
\text { sociaux }\end{array}$ & $\begin{array}{l}\text { citizen, society, community, family, senior, } \\
\text { youngster, child; désignation par l'áge (12-year-old } \\
\text { pupils) ou le métier (Joe the plumber)... }\end{array}$ \\
\hline 7 & Actualité & $\begin{array}{l}\text { drugs, medicare, welfare, tobacco, discrimination, } \\
\text { flum, marriage. ., financial crișs, inflattion, } \\
\text { unemployment, cut, wage, job, benefit ; oil, plant, } \\
\text { nuclear... }\end{array}$ \\
\hline 8 & Termes d'adresse & $\begin{array}{l}\text { prénoms et/ou noms de famille de chaque candidat, } \\
\text { taxèmes (senator governor, etc.), sir, Mr ; prénoms } \\
\text { des modérateurs (Jim...) et des journalistes } \\
\text { (Andrea...). }\end{array}$ \\
\hline 9 & $\begin{array}{l}\text { Langage courant et } \\
\text { informel (contexte } \\
\text { d'emploi non marqué) }\end{array}$ & $\begin{array}{l}\text { my friends, my folks, my fellow/foe, to muddle } \\
\text { through, kind of, the lot, to sound, to give a } \\
\text { confidence, thing, to look in the eye... . }\end{array}$ \\
\hline
\end{tabular}

Dans le cadre de la CDA, le genre «discours politique médiatisé » est considéré comme un genre complexe, intrinsèquement hybride, car il relève de quatre ordres de discours : l'ordre du système politique, l'ordre journalistique et médiatique, l'ordre des experts et l'ordre de la vie quotidienne. À chaque ordre correspond un ensemble de choix discursifs et rhétoriques : le discours des experts s'appuie, par exemple, sur des comparaisons, des relations de cause-conséquence, des prédictions, des faits quantifiés, etc.

Il apparaît que les catégories identifiées dans le tableau 3 peuvent être rattachées à un ou plusieurs de ces quatre ordres (tableau 4); l'appartenance des débats présidentiels au genre « discours politique médiatisé » s'en trouve confirmée. 
Tableau 4. Ordres de discours identifiés dans les débats présidentiels

\begin{tabular}{|l|l|}
\hline \multicolumn{1}{|c|}{ Ordre de discours } & \multicolumn{1}{c|}{ Traces lexicales } \\
\hline Ordre de discours du système politique & $\begin{array}{l}\text { lexique de la programmation politique + } \\
\text { référence à des institutions de la vie } \\
\text { politique, à des catégories } \\
\text { socioprofessionnelles, à des concepts } \\
\text { idéologiques, etc. }\end{array}$ \\
\hline $\begin{array}{l}\text { Ordre de discours du discours } \\
\text { journalistique médiatique }\end{array}$ & $\begin{array}{l}\text { référence à des sujets d'actualité + } \\
\text { référence à des lieux de conflits et à des } \\
\text { groupes sociaux / catégories } \\
\text { socioprofessionnelles + emploi de } \\
\text { termes d'adresse + citation de sources }\end{array}$ \\
\hline Ordre de discours de l'expertise & $\begin{array}{l}\text { référence à des sources externes + } \\
\text { emploi de termes précis (institutions, } \\
\text { sujets d'actualité) + quantification } \\
\text { (spatio-temporelle, de volume, etc.) }\end{array}$ \\
\hline Ordre de discours de la vie quotidienne & $\begin{array}{l}\text { langage courant et informel pour } \\
\text { désigner des événements, des } \\
\text { personnes, etc. }\end{array}$ \\
\hline
\end{tabular}

Les deux énoncés ci-dessous illustrent la façon dont les divers ordres de discours traversent la parole des candidats. Nous avons indiqué entre crochets [] les lexèmes et structures à rattacher à un (ou deux) ordre(s) de discours indiqué(s) en indice et encodé(s) de la manière suivante: SP renvoie à l'ordre du système politique, JM au discours journalistique médiatique, EX à l'ordre des experts et VQ à celui de la vie quotidienne.

[The CBO said $]_{\mathrm{EX} \text { ou JM }}$ very clearly that [if you were to adopt the [President $]_{\mathrm{SP}}$ 's $[\text { plan }]_{\mathrm{SP}}$, there would be $]_{\mathrm{EX}}$ a [trillion-dollar hole $]_{\mathrm{EX}}$ in [Social Security $]_{\mathrm{SP}}$, [because today's $[\text { workers }]_{\mathrm{SP}}$ pay in to the system for today's $\left.[\text { retirees }]_{\mathrm{SP}}\right]_{\mathrm{SP} \text { ou EX}}$. (J. Kerry, 2004)

Then $[y o u]_{\mathrm{JM}}$ have to $[\text { look at }]_{\mathrm{VQ}}$ our [proposals $]_{\mathrm{SP}}$ for our [economy $]_{\mathrm{SP}}$ not $[860$ billion dollars $]_{\mathrm{EX}}$ in new $[\text { spendings }]_{\mathrm{SP}}$, but for $[\text { the kinds of }]_{\mathrm{VQ}}[\text { reforms }]_{\mathrm{SP}}$ that $[\mathrm{keep}$ people in their jobs]VQ, get [middle-income Americans] $]_{\mathrm{SP}}$ working again, and [getting our [economy $]_{\mathrm{SP}}$ moving again $]_{\mathrm{VQ}}$. J. McCain, 2008)

Une telle conception des débats et de leur nature est cohérente avec l'idée selon laquelle ces événements ont à s'adapter aux supports d'expression ainsi qu'à l'évolution du contrat et du dispositif global de communication. Les changements sociétaux, les pressions socioculturelles, historiques et institutionnelles ont en effet influencé, et continuent d'influencer, les pratiques sociales et discursives des hommes politiques. K. H. Jamieson et D. Birdsell (1988) ont montré comment, dès le XIX ${ }^{e}$ siècle, les interventions politiques sont devenues de véritables spectacles et comment les candidats n'ont eu de cesse d'intégrer, en particulier au XX $\mathrm{XX}^{\mathrm{e}}$ siècle, les codes de divers supports de diffusion (presse écrite, radio, télévision) et de divers formats d'article ou d'émission (dépliants, communiqués, entretiens, émissions politiques ou talk-shows, débats). Les spécificités lexicales des candidats témoignent ainsi des changements auxquels les pratiques discursives se sont adaptées.

En outre, l'instance politique se conforme aux attentes de l'opinion publique, laquelle se veut prise en compte dans l'élaboration des politiques conduites et informée de l'état du pays, des décisions prises, des actions menées. Lors des campagnes électorales, les candidats cherchent à informer et séduire l'auditoire dans le but de l'amener à voter pour lui : informer-séduire-persuader, tel est le tryptique sous-jacent à chacune de leur 
prise de parole. En conséquence, chacun a, au cours des débats, à présenter et défendre son projet, prouver qu'il est capable d'affronter l'inconnu, de rassembler au-delà de son propre camp, de faire appliquer son programme ; il doit également séduire et fidéliser un auditoire toujours plus vaste et hétérogène du point de vue de l'origine sociale et professionnelle de ses membres, de leurs aspirations, de leurs attentes, etc. En d'autres termes, il doit «jouer" divers rôles, ce qui se traduit dans ses choix discursifs et rhétoriques: il est contraint de montrer qu'il possède et maîtrise (i) les codes du système politique : employer des lexèmes renvoyant par exemple à la programmation politique, aux institutions et aux codes législatifs régulant la société américaine ; (ii) les codes du monde médiatique (Rouveyrol 2004): traiter de sujets d'actualité et faire usage de termes d'adresse ; (iii) les codes du monde de l'expertise : décrire précisément et objectivement une situation, identifier des effets de cause-conséquence, se projeter dans le futur ; et (iv) les codes du citoyen moyen : parvenir à se construire l'image d'une personne accessible en employant des lexèmes relevant d'un langage courant. On notera à ce sujet que le recours à des syntagmes tels que [my] friends, folks, to look in the eye, muddle through, kind of, to sound rend les discours politiques moins formels, plus proches d'une conversation ordinaire: c'est le reflet d'une tendance relevée par N. Fairclough pour les discours publics et nommée "conversationnalisation » du discours (1994: 253).

L'imbrication des quatre ordres de discours observée dans les débats présidentiels permet donc de rendre compte de leur évolution dans le temps et de la multitude de rôles que les hommes politiques ont à jouer au cours d'une campagne électorale. Par ailleurs, l'approche de N. Fairclough permet de conforter l'idée selon laquelle les débats présentent des caractéristiques proches de celles des conversations: c'est ce que semblent indiquer les traces lexicales de l'ordre de discours de la vie quotidienne.

La nature du cadre interactif nommé « dialogue fictif » se voit également confirmée. La tension observée entre plan délocutoire (renvois à des référents externes aux échanges) et plan interlocutoire (adresses aux interlocuteurs) se retrouve au niveau des codes que les candidats empruntent au monde médiatique/journalistique et au milieu de l'expertise. Tandis que le premier est porté par la nécessité d'informer son auditoire des événements du monde et recourt tout autant à des référents relevant des sphères interlocutoire que délocutoire, seule la sphère délocutoire définit les comportements du milieu de l'expertise: ses acteurs s'intéressent en priorité à l'observation et à l'analyse de faits. On le voit, l'étude du genre ici menée permet d'éclairer la structuration lexicale des débats ainsi que la dynamique discursive qui les caractérise.

\section{Conclusion}

51 Dans cette étude, nous avons rappelé la place qu'occupent les débats présidentiels américains au sein des études en anglais de spécialité, et identifié certaines de leurs spécificités discursives, cognitives et génériques. Pour ce faire, nous avons emprunté à la logométrie sa méthodologie et ses outils de traitement statistique, ici, le logiciel Hyperbase. L'analyse de relevés lexicaux lemmatisés a tout d'abord confirmé l'idée selon laquelle les débats présidentiels, comme tout discours spécialisé, s'appuient sur un lexique tout à la fois commun et spécifique, lié au contexte politique et électoral. Ils partagent également avec le registre conversationnel certaines caractéristiques discursives, dont un flux de parole saccadé et un suremploi de données quantifiées. Les 
débats se définissent en outre par la construction d'un cadre dialogal dit «fictif » au sein duquel les relations candidats-auditoire sont moins marquées que celles entre candidats et événements/référents externes aux échanges. Cette observation a été confirmée par l'élaboration du cadre cognitif. Il est enfin apparu que les débats portent en eux les traces lexicales du genre "discours politique médiatisé », un genre intrinsèquement hybride.

Des questions relatives à la situation d'énonciation et au contrat de communication ont été envisagées à partir des relevés lexicaux. D'une part, il a été montré que le cadre cognitif élaboré par les candidats en discours est centré sur leur personne et la position $\mathrm{du}$ « ici-maintenant » afin que leur candidature ne fasse plus qu'un avec le statut de Président des États-Unis et que celle-ci apparaisse comme la réponse aux besoins du pays. D'autre part, il est apparu que les choix lexicaux témoignent de la variété des rôles assumés par les candidats en discours. Au cours d'un débat, ces derniers ne se contentent pas de maîtriser les termes spécialisés de leur domaine et de décrire leurs projets, ils se présentent tout à la fois comme des citoyens moyens, des politiciens, des experts et des hommes des médias. Une telle palette de rôles reflète une certaine adaptation à l'évolution des supports de diffusion et à l'élargissement de l'auditoire à convaincre.

Ainsi conduite, l'étude a fait converger analyses quantitatives et qualitatives pour voir émerger quelques-unes des spécificités langagières, discursives et culturelles des débats présidentiels américains : en ce sens, la démarche adoptée s'inscrit dans le cadre de l'anglais de spécialité.

\section{BIBLIOGRAPHIE}

Bendinelli, Marion. 2008. «Contribution à l'étude des modalités intersubjectives au sein d'un corpus de débats présidentiels médiatisés aux États-Unis ». Mémoire de master sous la codirection de Sylvie Mellet (DR - CNRS) et de Laurent Rouveyrol (MCF). Université Nice SophiaAntipolis.

Biber, Douglas, Stig Johansson, George Leech, Susan Conrad et Edward Finegan. 1999. Grammar of Spoken and Written English. Londres : Longman.

Bommier-Pincemin, Bénédicte. 1999. «Diffusion ciblée automatique d'informations : conception et mise en œuvre d'une linguistique textuelle pour la caractérisation des destinataires et des documents ». Thèse de Doctorat en linguistique, Université Paris 4 Sorbonne, chapitre VII : «Caractérisation d'un texte dans un corpus : du quantitatif vers le qualitatif », § A. « Définir un corpus », 415-427. Consulté le 24 juin 2011 <http://www.revue-texto.net/Inedits/Pincemin/PDF/ Chapitre/chap7.pdf>.

Bourdieu, Pierre. 1982. Ce que parler veut dire :l'économie des échanges linguistiques. Paris : Fayard. Charaudeau, Patrick. 2002. «Contrat de communication ». In Charaudeau, Patrick et Dominique Maingueneau (dir.), Dictionnaire d'analyse du discours. Paris : Seuil, 138-41. 
Charaudeau, Patrick. 2005. Le Discours politique. Les Masques du pouvoir. Paris : Librairie Vuibert éd. Chilton, Paul A. 2004. Analysing Political Discourse: Theory and practice. Londres : Routledge.

Dailey, William O., Edward A. Hinck et Shelly S. Hinck. 2008. Politeness in Presidential Debates Shaping Political Face in Campaign Debates from 1960 to 2004. Lanham, MD : Rowman \& Littlefield Publishers, Inc.

Deyrich,Marie-Christine. 2004. « Exploration didactique de la langue du milieu professionnel à l'université : quel apport pour la définition de tâches d'enseignement-apprentissage ? ». ASp 43-44, 125-134. Consulté le 24 juin 2011 <http://asp.revues.org/1106>.

Duchastel, Jules et Victor Armony. 1993. « Un protocole de description de discours politiques ». In École Nationale Supérieure de Télécommunications, Actes des Secondes journées internationales d'analyse statistique de données textuelles, Paris : Télécom, 159-183. Consulté le 24 juin 2008 <http:// www.ling.uqam.ca/sato/publications/bibliographie/Jul10.htm>

Fairclough, Norman. 1994. "Conversationalization of public discourse and the authority of consumer ». In Keat, Russell, Nigel Whiteley et Nicholas Abercrombie, The Authority of the Consumer. Londres : Routledge, 254-268.

Fairclough, Norman. 1995. Media Discourse. Londres : Edward Arnold.

Fairclough, Norman. 2000. «Discourse, Social Theory, and Social Research: The Discourse of Welfare Reform ». Journal of Sociolinguistics 4/2, 163-195.

Fairclough, Norman. 2001. « The dialectics of discourse ». Textus 14/2, 231-242.

Fauconnier, Gilles. 1985. Mental Spaces: Aspects of meaning construction in natural language. Cambridge, MA : MIT Press.

Friedenberg, Robert V. (dir.). 1994. Rhetorical Studies of National Presidential Debates - 1960-1992. Westport, CT : Praeger Publishers.

Friedenberg, Robert V. (dir.). 1997. Rhetorical Studies of National Presidential Debates - 1996. Westport, CT : Praeger Publishers.

Jamieson, Kathleen H. et David S. Birdsell. 1988. Presidential Debates: The Challenge of Creating an Informed Electorate. New-York : Oxford University Press.

Kerbrat-Orecchioni, Catherine. 1990. Les Interactions verbales. Tome I. Paris : Armand Colin.

Mayaffre, Damon. 2004. Paroles de président. Jacques Chirac (1995-2003) et le discours présidentiel sous la Ve République. Paris : Champion.

Minow, Newton N. et Craig L. Lamay. 2008. Inside the Presidential Debates: Their Improbable Past and Promising Future. Chicago : University of Chicago Press.

Petit, Michel. s-d. Notes de position sur les notions de « spécialité », « milieu spécialisé » et « anglais de spécialité ». Consultées le 24 juin $2011<$ http://www.langues-vivantes.ubordeaux2.fr/frsa/pagesperso/michelpetit/recherchemp.html>.

Petit, Michel. 2004. «Quelques réflexions sur la fiction à substrat professionnel : du général au particulier ». In Petit, Michel et Shaeda Isani (dir.), Aspects de la fiction à substrat professionnel, Bordeaux, Université Bordeaux 2, 3-23. Consulté le 24 juin 2011 <http://www.langues-vivantes.ubordeaux2.fr/frsa/pagesperso/michelpetit/pdf/faspAvantPropos.pdf>.

Petit, Michel. 2010. « Le discours spécialisé et le spécialisé du discours : repères pour l'analyse du discours en anglais de spécialité », E-rea [En ligne], 8/1. Consulté le 29 août $2011<$ http:// erea.revues.org/1400>. 
Rouveyrol, Laurent. 2003. «Étude pragmatique de la variation linguistique dans le débat politique médiatisé en anglais. Les modalités et marques d'implications du locuteur dans le discours ». Thèse de doctorat sous la direction de Françoise Dubois-Charlier, Université d'Aix-enProvence.

Rouveyrol, Laurent. 2004. «Des milieux des uns aux discours des autres : à propos d'interactions médiatiques ». ASp 45-46, 43-59. Consulté le 24 juin 2011 <http://asp.revues.org/853>.

Société des Anglicistes de l'Enseignement Supérieur (SAES). 2011. «Évolution et enjeux des formations et de la recherche dans le secteur », document élaboré et approuvé par la Commission Formations de la SAES le 28 janvier 2011. Consulté le 24 juin $2011<\mathrm{http}$ ://sha.univ-poitiers.fr/ saesfrance/spip.php?article262>.

Trognon Alain et Janine Larrue. 1994. Pragmatique du discours politique. Paris : Armand Colin.

Verschueren, Jef. 1999. Understanding Pragmatics. Londres : Edward Arnold.

Vion, Robert. 1992. La communication verbale. Analyse des interactions. Paris : Hachette.

Ressources électroniques (sites consultés le 29 juin 2011)

Archives audiovisuelles de John Fitzgerald Kennedy. <www.jfklibrary.org>.

C-Span Channel - archives audiovisuelles. < http://www.c-spanvideo.org/videoLibrary>.

Centre National de Ressources Textuelles et Lexicales (CNRS - UMR Atilf). <www.cnrtl.fr>.

Commission of Presidential Debates. <www.debates.org>.

Corpus of Contemporary American English. <www.americancorpus.org>.

Étiqueteur morpho-syntaxique TreeTagger. <http://www.ims.uni-stuttgart.de/projekte/ corplex/TreeTagger/DecisionTreeTagger. html>.

League of Women Voters. <www.lwv.org>.

\section{Logiciel de traitement statistique}

Hyperbase (version 9 - avril 2011), logiciel documentaire et statistique pour la création et l'exploitation de bases hypertextuelles, développé par Etienne Brunet, professeur émérite, Laboratoire « Bases, Corpus, Langage » - UMR 6039 - Université Nice Sophia-Antipolis - CNRS. Documentation et version d'évaluation disponibles en ligne <http://ancilla.unice.fr>. Site du laboratoire <http://www.unice.fr/bcl>.

\section{NOTES}

1. Le logiciel de traitement documentaire et statistique Hyperbase a été mis au point par Étienne Brunet, professeur émérite à l'Université Nice Sophia-Antipolis, laboratoire «Bases, Corpus, Langage » - UMR 6039. Hyperbase permet non seulement d'exploiter le contenu lexical (formes graphiques et lemmes), grammatical et syntaxique du corpus considéré, mais aussi de s'intéresser à ses segments répétés, collocations et réseaux de co-occurrents. On se reportera au manuel d'utilisation pour de plus amples détails (référence en bibliographie).

2. On retrouve ici la distinction effectuée en anglais entre "political discourse", le discours politique, et "political speeches", syntagme qui renvoie aux prises de parole observées dans le domaine de la politique.

3. Le terme " professionnel » est sujet à débat: il est ici entendu comme étant lié à l'exercice d'une profession, une activité régulière et rémunérée, permettant de classer les individus dans 
une catégorie sociale reconnue par l'administration (dictionnaire CNRTL en ligne). Une acception plus large peut également être adoptée : voir à ce sujet les notes de position de Michel Petit (références en bibliographie).

4. Les débats à trois candidats des années 1980 et 1992 ont en effet été éliminés du corpus existant pour garantir l'homogénéité du corpus d'étude, notamment en ce qui concerne le dispositif de communication. Pour une définition de corpus d'étude, voir B. Bommier-Pincemin (1999 : 419-20).

5. En anglais, ces scénographies (ou formats) sont désignées sous le nom de «press-conference type ", "town-hall meeting " et "conversation type " - voir notamment à ce sujet Jamieson et Birdsell (1988) et Minow et Lamay (2008).

6. La lemmatisation est effectuée en amont de l'implémentation des fichiers textuels au moyen de TreeTagger, outil développé par Helmut Schmidt à l'Université de Stuttgart.

7. Ces contraintes sont liées à l'emploi d'une langue donnée, ici l'anglais ; elles transcendent les questions de domaine de spécialité et de genre discursif. On relève ainsi parmi les 20 premiers rangs les articles the et $a$, les auxiliaires be, have et do et les prépositions to, of et in.

8. Les lemmes year,time, thing,way occupent respectivement les rangs 46, 68, 84 et 92 dans notre corpus et les rangs 54, 52, 97 et 84 dans COCA.

9. Les lemmes people, president, country, tax, senator, world et government occupent respectivement les rangs 32, 37, 57, 59, 83, 89 et 99 dans notre corpus et les rangs 62, 304, 166, 510, 2794, 123 et 201 dans COCA. Les lemmes dollar et America n'apparaissent pas dans la liste des 5000 lemmes les plus fréquents du corpus COCA.

10. Par "phrase clé », on entend une phrase relevée par le logiciel Hyperbase parce qu'elle réunit certaines des spécificités lexicales et/ou grammaticales (formes graphiques ou lemmes se présentant comme suremployés) de la partition envisagée, ici les locuteurs.

11. Dans le corpus COCA, $70 \%$ des occurrences du pronom $I, 60 \%$ de celles de we et près de $50 \%$ des occurrences de not et n't sont employées dans des productions orales, authentiques ou fictives.

12. Notre traduction

13. Notre traduction

14. Notre traduction

15. Nous avons pu avoir accès aux conseils donnés jusqu'en 2000 par les conseillers en communication (ou spin doctors) au travers des travaux de R. Friedenberg $(1994,1997)$ : de telles indications ne semblent pas avoir été données. Il n'est malheureusement pas (encore) possible de le déterminer pour les débats plus récents.

16. Les pronoms de troisième personne représentent plus du double des pronoms de deuxième personne (8 644 contre 3254 occurrences).

17. Le modèle original de Paul A. Chilton est constitué de trois espaces, les espaces géographique et temporel étant confondus en un seul espace «spatio-temporel ». Nous faisons le choix de les distinguer car il nous semble que la représentation gagne ainsi en précision. De plus, tel qu'il est conçu, l'espace modal ne permet pas de faire apparaître les diverses valeurs (épistémique, déontique, etc.) des expressions modales. C'est une nécessité qu'il nous faut encore modéliser : dans le cadre du présent travail, nous préférons par conséquent ne pas exploiter l'axe modal.

18. L'espace modal n'est pas complété pour les raisons invoquées en note 17. Les parenthèses indiquent que ces lexèmes peuvent recevoir une position quelque peu différente en fonction de la façon dont le candidat les emploie en discours : en effet, la distance au locuteur peut varier selon qu'il les utilise pour se valoriser ou pour délégitimer son adversaire (Bendinelli 2008).

19. Le choix de la CDA est conditionné par le fait que notre réflexion s'inscrit dans le cadre de l'analyse de discours politiques. Pour un panorama plus complet des études portant sur la notion de genre, il serait intéressant d'éclairer la définition formulée par Norman Fairclough par celles de Patrick Charaudeau pour son point de vue communicationnel, de John M. Swales parce que ses 
travaux portent sur les discours de spécialité (notamment scientifiques) et de Vijay K. Bhatia qui s'intéresse, entre autres, aux phénomènes d'imbrication de genres. Cette dernière réflexion trouve un écho particulier avec l'analyse présentée en section 5.2.

20. Notre traduction

21. L'expression «mise en texte » est une traduction de «texturing ", notion renvoyant à celle de «texture » que N. Fairclough emprunte à la linguistique systémique fonctionnelle de Michael A.K. Halliday.

22. Pour une présentation plus détaillée de la CDA, voir Rouveyrol (2003 : 55-67).

\section{RÉSUMÉS}

Cet article propose d'inscrire l'étude des débats présidentiels américains dans le champ de l'anglais de spécialité et d'y envisager la contribution d'analyses quantitatives empruntées au cadre théorique et méthodologique de la logométrie (Mayaffre 2004). Le corpus d'étude, composé des 22 face-à-face à deux candidats organisés depuis 1960, est traité à l'aide du logiciel Hyperbase. Le relevé lemmatisé des hautes fréquences lexicales montre que les débats sont, sur le plan grammatical, discursif et interactionnel, sensiblement proches du registre conversationnel. Il permet également d'en reconstruire le cadre cognitif (Chilton 2004) et de souligner l'importance donnée aux relations intersubjectives au sein de ce cadre. L'étude des spécificités lexicales des candidats met par ailleurs en évidence le caractère intrinsèquement hybride des pratiques discursives des candidats et confirme l'appartenance des débats présidentiels au genre «discours politique médiatisé » (Fairclough 1995). Cette étude, mêlant analyses qualitatives et quantitatives, contribue à montrer que la logométrie peut outiller la recherche en anglais de spécialité en mettant au jour la structuration discursive, cognitive et générique d'un discours spécialisé.

This paper addresses the question of American presidential debates from the ESP viewpoint and considers how linguistic quantitative analyses may help to identify some of their characteristics. The corpus consists of 22 debates (only face-to-face encounters) and covers eight election years since 1960. The quantitative analyses lie within the framework of logometry (Mayaffre 2004) and are carried out with the software Hyperbase; in particular, they are grounded on the lemma frequency lists of the corpus and on the lexical specificities of the candidates. The studies show that presidential debates share some discursive and interactional similarities with conversations and belong to a hybrid discourse genre (Fairclough 1995). They also indicate that intersubjective relations are of particular importance within the cognitive frame (Chilton 2004) of the debates. The paper highlights the fact that linguistic quantitative analyses may contribute to ESP research since they help to study discourse phenomena from several linguistic perspectives.

\section{INDEX}

Keywords : American presidential debate, English for Specific Purposes, linguistic quantitative analysis, logometry, specialised discourse

Mots-clés : analyse linguistique quantitative, anglais de spécialité, débat présidentiel américain, discours politique, discours spécialisé, logométrie 


\section{AUTEUR}

\section{MARION BENDINELLI}

Marion Bendinelli est doctorante à l'Université de Nice Sophia-Antipolis où elle est Attachée Temporaire d'Enseignement et de Recherche en linguistique générale. Elle appartient à l'UMR 6039, « Bases, Corpus, Langage ». Ses travaux portent sur l'expression de la modalité en anglais dans le cadre de la communication persuasive, et en particulier, au sein des débats présidentiels américains. Ses questions de recherche se situent dans le champ de l'analyse de discours politique, de la logométrie, de la linguistique énonciative et de la linguistique interactionnelle. mbendinelli@hotmail.fr 